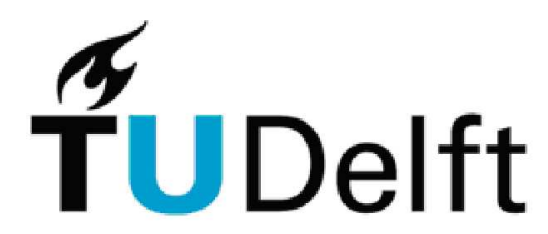

TU Delft Library

Prometheusplein 1

Postbus 98

2600 MG Delft

Tel: +31 (0) 152785678

Fax: +31 (0) 152785706

Email: library@tudelft.nl

www.library.tudelft.nl

Aan: T.N.O.

RIS / LIDY-MARIE OUWEHAND - KMR 3C03

POSTBUS 96864

2509 JG DEN HAAG

NEDERLAND

Aanvraag nr: 1663692

Uw referentie(s): TS41021031 - H

Artikelomschrijving:

Aantal kopieën: $\mathbf{5}$

Artikel: THIN FILM THERMISTOR WITH POSITIVE TEMPERATURE COE

Auteur: LENZ

Titel: $\quad$ APPLIED PHYSICS LETTERS

Jaar:

2016

Vol. 109

Nr. 13

Pag. 133302

Plaatsnummer: via:http://aip.scita 
Thin film thermistor with positive temperature coefficient of resistance based on phase separated blends of ferroelectric and semiconducting polymers

Thomas Lenz, , Hamed Sharifi Dehsari, Kamal Asadi, Paul W. M. Blom, Wihelm A. Groen, and Dago M. de Leeuw

Citation: Appl. Phys. Lett. 109, 133302 (2016); doi: 10.1063/1.4963349

View online: $h$ ttp://dx.dol.org/10.1063/1.4963349

View Table of Contents: http://aip.scitation.org/toc/apl/109/13

Published by the American Institute of Physics 


\title{
Thin film thermistor with positive temperature coefficient of resistance based on phase separated blends of ferroelectric and semiconducting polymers
}

\author{
Thomas Lenz, ${ }^{1,2, a)}$ Hamed Sharifi Dehsari, ${ }^{1}$ Kamal Asadi, ${ }^{1}$ Paul W. M. Blom, ${ }^{1,2}$ \\ Wilhelm A. Groen, ${ }^{3,4}$ and Dago M. de Leeuw ${ }^{1,3}$ \\ ${ }^{1}$ Max-Planck Institute for Polymer Research, Ackermannweg 10, 55128 Mainz, Germany \\ ${ }^{2}$ Graduate School Materials Science in Mainz, Staudinger Weg 9, 55128 Mainz, Germany \\ ${ }^{3}$ Faculty of Aerospace Engineering, Delft University of Technology, Kluyverweg 1, \\ 2629 HS Delft, The Netherlands \\ ${ }^{4}$ Holst Centre, P.O. Box 8550,5605 KN Eindhoven, The Netherlands
}

(Received 24 May 2016; accepted 13 September 2016; published online 27 September 2016)

\begin{abstract}
We demonstrate that ferroelectric memory diodes can be utilized as switching type positive temperature coefficient (PTC) thermistors. The diode consists of a phase separated blend of a ferroelectric and a semiconducting polymer stacked between two electrodes. The current through the semiconducting polymer depends on the ferroelectric polarization. At the Curie temperature the ferroelectric polymer depolarizes and consequently the current density through the semiconductor decreases by orders of magnitude. The diode therefore acts as switching type PTC thermistor. Unlike their inorganic counterparts, the PTC thermistors presented here are thin film devices. The switching temperature can be tuned by varying the Curie temperature of the ferroelectric polymer. Published by AIP Publishing. [http://dx.doi.org/10.1063/1.4963349]
\end{abstract}

Thermistors with a positive temperature coefficient (PTC) of the resistance are divided into two groups according to their operation mechanism and materials used. The first group uses doped silicon or a metal. It exhibits a linear resistance-temperature curve with a small PTC and is typically used as temperature sensors. The second group of PTC thermistors is the switching type. It shows highly nonlinear resistance-temperature curves and, therefore, is widely used in electrical engineering as self-regulating heating elements and as current limiter for overcurrent protection. ${ }^{1-3} \mathrm{~A}$ wellknown example is a resettable fuse that consists of a composite of conductive fillers, such as carbon black, embedded in an insulating polymer matrix, such as polyethylene. ${ }^{4-10} \mathrm{At}$ room temperature the conductive particles form a percolating path and the resistance is low. ${ }^{11-13}$ When the temperature increases, the large volume expansion of the semi-crystalline polymer close to its melting point breaks up the percolation path and the resistance dramatically increases.

The most commonly used switching type PTC, however, is based on ferroelectric doped $\mathrm{BaTiO}_{3}$ ceramics. ${ }^{14-18}$ Undoped $\mathrm{BaTiO}_{3}$ is an insulator and as such cannot be used as a PTC thermistor. To that end, $\mathrm{BaTiO}_{3}$ is $n$-type doped by replacing $\mathrm{Ba}$ with a trivalent donor such as $\mathrm{La}$ or by replacement of $\mathrm{Ti}$ by pentavalent $\mathrm{Nb} .{ }^{19,20}$ The electron traps at the grain boundaries dominate the electrical transport and the PTC effect. Above the Curie temperature, the dielectric constant decreases according to Curie-Weiss law, which causes the potential barrier for electrons at the grain boundary to increase. Consequently, the resistance increases dramatically.

The commercial switching type PTCs are typically bulky, made as discrete component or as surface mounted devices. The manufacturing process of densely sintered, ceramic $\mathrm{BaTiO}_{3}$ requires careful control of materials and impurities. Contaminations in the order of a few ppm can

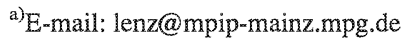

cause major changes in the thermal and electrical properties. Thin film thermistors are elusive; the high process temperature leads to intermixing and strain caused by the substrate can suppress the phase transition. ${ }^{21}$ Finally, the operational mechanism relies on the resistance modulation of grain boundaries, which cannot easily be transferred to thin films. Here we demonstrate a switching type PTC thermistor that can be processed as a thin film. Additional advantages are processing at low temperature from solution and tunability of the transition temperature.

The operational mechanism is based on the decrease of the ferroelectric polarization at the Curie temperature. $\mathrm{P}(\mathrm{VDF}-\mathrm{TrFE})$ is an insulator and cannot be electrically doped like $\mathrm{BaTiO}_{3}$. To utilize the change in polarization, we use a ferroelectric diode that consists of a phase separated blend film of a ferroelectric and a semiconducting polymer sandwiched between two electrodes. ${ }^{22,23}$ The electrodes are selected such that they form a Schottky contact with the semiconductor. Consequently, the current density is injection limited and low. However, by polarizing the ferroelectric polymer the injection barrier is eliminated leading to an Ohmic contact and a high space charge limited current density through the organic semiconductor. However, at the Curie temperature, $T_{c}$, the ferroelectric polymer depolarizes. The injection barrier cannot be overcome and, consequently, the current density drops. Effectively, the resistance dramatically increases with temperature. We show that a thin film switching type PTC thermistor is realized. By using a welldefined poling protocol the change in resistance is reversible and the switching temperature can be set by the Curie temperature of the ferroelectric polymer.

As a ferroelectric polymer we used the random copolymer of vinylideneffuoride with trifluoroethylene $\mathrm{P}(\mathrm{VDF}-\mathrm{TrFE}){ }^{24-26}$ Batches with different ratios of VDF and TrFE were purchased from Solvay. The ferroelectric properties were characterized in capacitors. Thin films of about $300 \mathrm{~nm}$ were fabricated by 


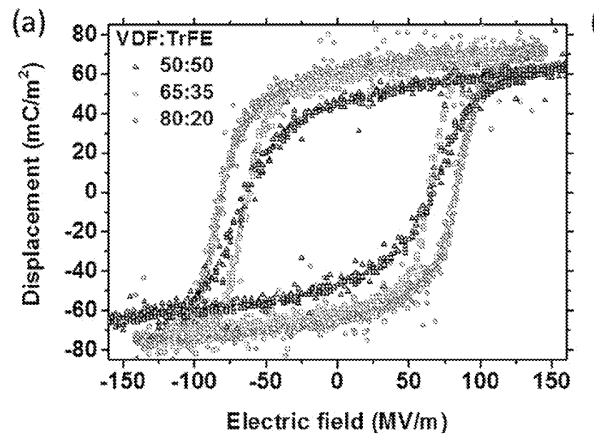

(b)

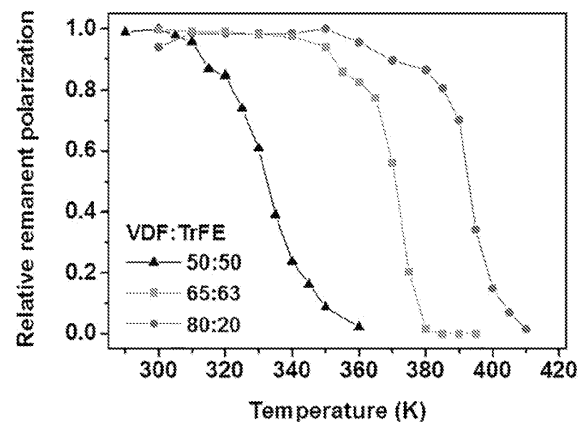

FIG. 1. (a) Electric displacement-electric field loops for ferroelectric capacitors with different composition of the copolymer P(VDF-TrFE) measured with a Sawyer-Tower setup. The legend shows the composition. (b) Extracted remanent polarization of the capacitors of (a) as a function of temperature. The lines are guide to the eye.
TABLE I. Extracted ferroelectric parameters of P(VDF-TrFE) with different composition. Values are listed for the remanent polarization, $P_{r}$, the coercive field, $E_{C}$, and the Curie temperature, $T_{C}$, obtained from hysteresis loops, from Differential Scanning Calorimetry (DSC) measurements and from literature reports. ${ }^{30-32}$ DSC measurements were performed with a Mettler-Toledo $822 / 400$ DSC with a modular measurement unit using a heating rate of $10 \mathrm{~K} / \mathrm{min}$ up to $200^{\circ} \mathrm{C}$.

\begin{tabular}{lccccc}
\hline VDF:TrFE & $P_{\mathrm{r}}\left(\mathrm{mC} / \mathrm{m}^{2}\right)$ & $\mathrm{E}_{\mathrm{c}}(\mathrm{MV} / \mathrm{m})$ & $\mathrm{T}_{\mathrm{c}}(\mathrm{K})$ & $\mathrm{T}_{\mathrm{c}}(\mathrm{DSC})$ & $\mathrm{T}_{\mathrm{c}}$ (Lit) \\
\hline $50: 50$ & 47 & 65 & 332 & 337 & 331 \\
$65: 35$ & 61 & 64 & 371 & 378 & 368 \\
$80: 20$ & 65 & 82 & 393 & 400 & 397 \\
\hline
\end{tabular}

wire-bar coating from $4 \mathrm{wt} . \%$ solutions in cyclohexanone. Substrate temperature was chosen as $80^{\circ} \mathrm{C}$ to minimize the surface roughness. ${ }^{27,28}$ Subsequently, films were annealed at $140^{\circ} \mathrm{C}$ for $2 \mathrm{~h}$ in vacuum to enhance the crystallinity of $\mathrm{P}(\mathrm{VDF}-\mathrm{TrFE})$ and, hence, to enhance its ferroelectric properties. ${ }^{29}$ Ferroelectric hysteresis loops of comprising capacitors are presented in Fig. 1(a). The values extracted for the remanent polarization, viz. displacement at zero electric field, and the coercive field, viz. electric field at zero displacement, are listed in Table I. We observe an increase of remanent polarization with increasing VDF content in agreement with literature. $^{25}$ The relative remanent polarization decreases with increasing temperature as shown in Fig. 1(b). We arbitrarily extracted $T_{c}$ as the temperature where the relative remanent polarization has decreased by $50 \%$. With increasing content of VDF, $T_{c}$ shifts to higher temperatures. The values determined for $T_{c}$ are confirmed with Differential Scanning
Calorimetry (DSC) measurements, where $T_{c}$ was obtained from the maximum of the ferroelectric-paraelectric transition peak in the second heating cycle. The values shown in Table I are in good agreement with literature reports. ${ }^{30-32}$

PTC thermistors were realized by combining ferroelectric $\mathrm{P}(\mathrm{VDF}-\mathrm{TrFE})$ with the semiconducting polymer poly $(9,9-$ dioctylfluorene) (PFO), purchased from TNO/Holst Centre. P(VDF-TrFE) and PFO were dissolved in cyclohexanone in a ratio 9:1. The blend solution of $4 \mathrm{wt}$ \% was wire-bar coated at $80^{\circ} \mathrm{C}$ on glass substrates comprising bottom electrodes of $50 \mathrm{~nm} \mathrm{Au}$ with a $2 \mathrm{~nm} \mathrm{Cr}$ adhesion layer followed by annealing at $140^{\circ} \mathrm{C}$ for $2 \mathrm{~h}$. The film thickness amounted to about $300 \mathrm{~nm}$. An Atomic Force Microscopy (AFM) height image of a resulting blend film is presented in Fig. 2(a). The surface roughness amounts to about $10 \mathrm{~nm}$. The inset of Fig. 2(b) shows a schematic cross-section. The microstructure exhibits the characteristic morphology of a phase separated blend and consists of bicontinuous columnar PFO domains embedded in the P(VDFTrFE) matrix as experimentally verified by synchrotron $\mathrm{X}$-ray measurements $^{33}$ and by scanning X-ray spectro-microscopy measurements (STXM). ${ }^{34}$ The needle-like morphology of the matrix is typical for semi-crystalline P(VDF-TrFE). ${ }^{24,35}$

Fabrication of PTC thermistors was finished by thermal evaporation of $\mathrm{Au}$ top electrodes yielding a device area of about $0.16 \mathrm{~mm}^{2}$. The current voltage characteristics measured at ambient temperature are presented in Fig. 2(b).

The top Au contact is grounded and we sweep the bottom Au electrode from $0 \mathrm{~V}$ to $+20 \mathrm{~V}$. The Au-PFO Schottky contact is injection limited. At low bias the current density is
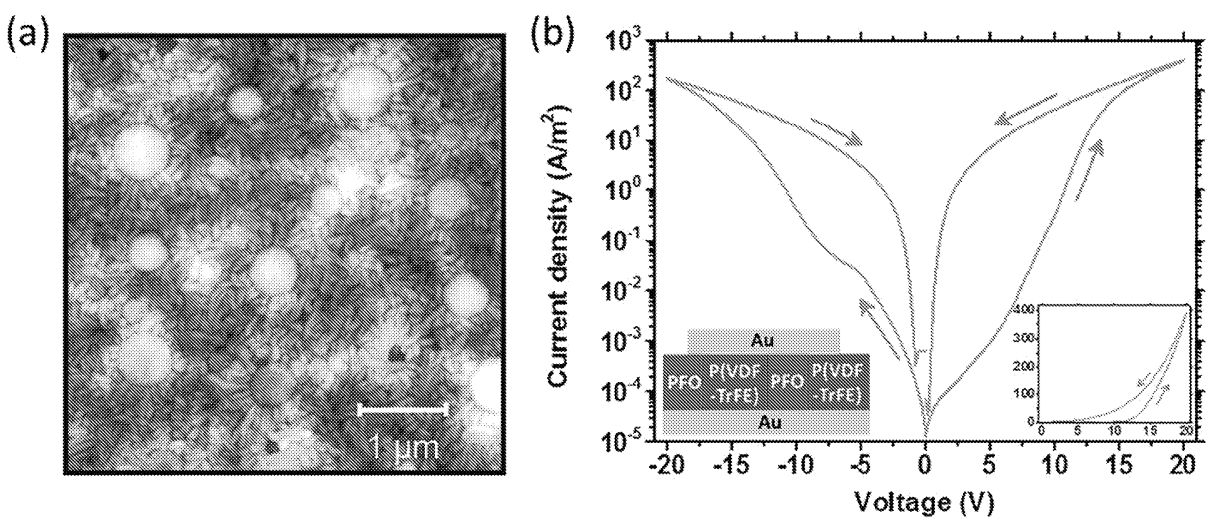

FIG. 2. (a) AFM micrograph (height scale of $70 \mathrm{~nm}$ ) of a phase-separated blend film of PFO and P(VDF-TrFE) (65:35). Measurements were performed using a Nanoscope Dimension 3100 (Bruker). The film thickness as determined with a DEKTAK surface profilometer amounted to about 300 nm. (b) Current density as a function of applied electric field at ambient temperature for the comprising memory diode. The measurements were performed in dynamic vacuum of $10^{-6}$ mbar using a $4155 \mathrm{~B}$ Semiconductor Parameter Analyzer (Agilent). The left inset shows a schematic cross-section of the thermistor illustrating the bicontinuous domains of PFO embedded in a matrix of P(VDF-TrFE). The right inset shows the current density for positive bias in a linear scale. 

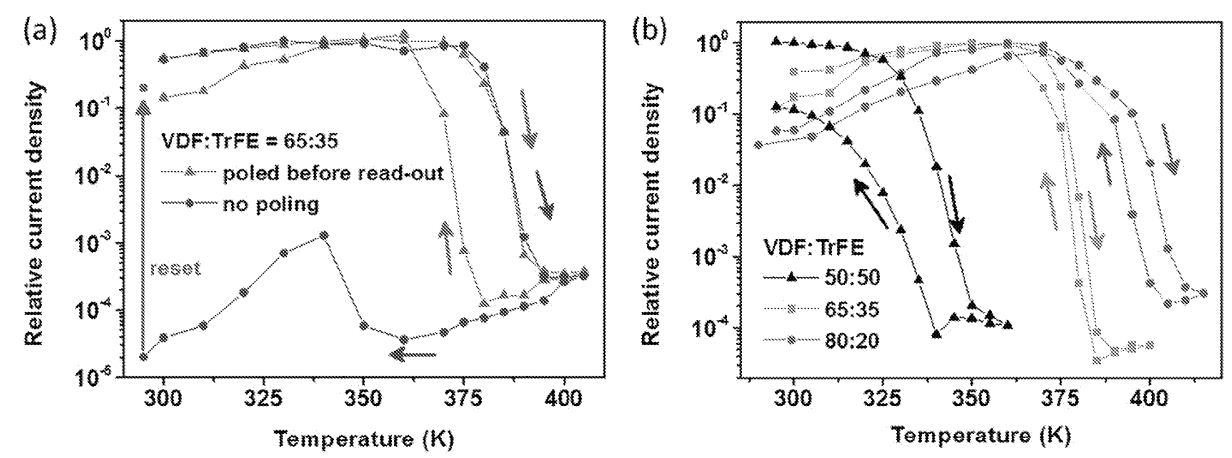

FIG. 3. (a) Current density of a ferroelectric diode as a function of temperature. The diode was poled in the On-state at ambient temperature and the current density at $+4 \mathrm{~V}$ was measured as a function of temperature up to $400 \mathrm{~K}$ and back (blue dots, lines are guide to the eye). The red triangles represent the current density at $+4 \mathrm{~V}$ after poling the diode $+20 \mathrm{~V}$ for each temperature. In this case, a reversible PTC behavior is obtained. The arrows indicate the sequence of the measurements. (b) Reversible PTC characteristics of thermistors with different composition of P(VDF-TrFE). Current density was again measured at $+4 \mathrm{~V}$ after poling at $+20 \mathrm{~V}$. The transition temperatures correspond to the Curie temperature as can be inferred from Fig. $1(\mathrm{~b})$.

low; the diode is in the Off-state. However, by increasing the positive bias, the ferroelectric P(VDF-TrFE) gets fully polarized at the coercive voltage, here around $10 \mathrm{~V}$. The stray electric field between the polarization charges and the compensating charges in the electrode then effectively eliminates the injection barrier. The contact becomes Ohmic ${ }^{36}$ and the current density increases by orders of magnitude. The diode is in the On-state. The ferroelectric polarization does not change upon sweeping back to $0 \mathrm{~V}$. Hence, the diode remains in the On-state. Since the diode is symmetric, we observe a comparable curve for negative bias, where the top $\mathrm{Au}$ electrode is the injecting contact. As the bistable diode can reversibly be switched between the On-state and the Off-state it has been used as a non-volatile bistable memory. $22,37-45$

Here, in order to realize a PTC thermistor, we make use of the temperature dependence of the On-state current density. We first poled the thermistor at ambient temperature in the On-state. Then, we measured the current density at $+4 \mathrm{~V}$, while gradually increasing the temperature up to $400 \mathrm{~K}$ and back, as indicated by the blue dots in Fig. 3(a). The blue arrows indicate the sequence of the measurements. The current density remains constant up to the $T_{c}$ of $\mathrm{P}(\mathrm{VDF}-\mathrm{TrFE})$ (65:35). Due to the depolarization of the ferroelectric, the diode then switches to the Off-state and the current density drops by orders of magnitude. Upon cooling down the diode stays in the Off-state and the current density stays low, because the ferroelectric remains unpolarized. We repeatedly observed an anomalous increase of the Off current density at around $335 \mathrm{~K}$. We note that a phase transition of PVDF at that temperature has been reported by dilatometry. ${ }^{46}$ The microscopic origin is as yet unknown.

The diode behaves as a fuse and can be reset by poling at room temperature as indicated by the light blue arrow in Fig. 3(a). In order to obtain reversible PTC behavior the conducting state has to be recovered at each temperature. This can be achieved by poling the diode before each read-out.

The red triangles in Fig. 3(a) represent the current density at $+4 \mathrm{~V}$ after poling the diode at $+20 \mathrm{~V}$ for each temperature. The poling procedure itself, such as the pulse time and length, is not critical. The curves for heating and cooling coincide meaning that the PTC behavior is indeed reversible. The transition temperature corresponds to the Curie temperature as can be concluded from Fig. 1(b). This shows that the origin of the switching type PTC behavior is the ferroelectric-paraelectric transition of $\mathrm{P}(\mathrm{VDF}-\mathrm{TrFE})$ at the Curie temperature. This is confirmed by varying the composition of the ferroelectric copolymer P(VDF-TrFE). In Fig. $3(b)$ the current density at $+4 \mathrm{~V}$ is plotted as a function of temperature for different VDF:TrFE ratios. For each measurement the diode was first poled at $+20 \mathrm{~V}$. Reversible PTC behavior is obtained. The transition temperature increases with VDF content, which corresponds to the increase of $T_{c}$, as can be inferred from Fig. 1(b) and Table I. The hysteresis in the measurements is similar to that observed in the DSC measurements and can be minimized by adjusting the processing conditions and the poling protocol. For example, the smaller hysteresis for VDF:TrFE 65:35 in Fig. 3(b) (orange curve) as compared with Fig. 3(a) (red curve) is realized using a larger poling time.

The PTC concept presented here works as long as the microstructure consists of bicontinuous columnar domains of PFO embedded in a matrix of ferroelectric P(VDF-TrFE). Experimentally, we did verify this microstructure in thin films with a thickness up to $500 \mathrm{~nm}$. The resistance scales with the thickness. But as the Curie temperature is not changing with thickness, the value of the temperature coefficient is thickness-independent.

In summary, we have demonstrated a switching type PTC thermistor. The robust thin film PTC is based on a bistable ferroelectric diode, where the current density through the semiconductor is governed by the polarization of the ferroelectric polymer. The operational mechanism of the thermistor relies on the ferroelectric-to-paraelectric transition of the ferroelectric copolymer at the Curie temperature. Upon depolarization of the ferroelectric polymer at the Curie temperature, the current density of the thermistor exhibits a non-linear decrease. The decrease of the current density, which is equivalent to an increase of the resistance of the thermistor, is reversible proving PTC behavior. We have shown that the switching temperature of the thin film PTC thermistor can be tuned by varying the composition of the ferroelectric copolymer, since the composition itself determines the Curie temperature.

We gratefully acknowledge the technical support from C. Bauer and F. Keller from the Max Plank Institute for 
Polymer Research, Mainz, Germany. Author K.A. acknowledges the Alexander von Humboldt Foundation for the funding provided in the framework of the Sofja Kovalevskaja Award, endowed by the Federal Ministry of Education and Research, Germany. Author T.L. acknowledges financial support by the Graduate School Materials Science in Mainz.

${ }^{1}$ Y. L. Chen and S. F. Yang, Adv. Appl. Ceram. 110, 257 (2011).

${ }^{2}$ T. R. Shrout, D. Moffatt, and W. Huebner, J, Mater. Sci. 26, 145 (1991).

${ }^{3}$ F. A. Doljack, IEEE Trans. Compon., Hybrids, Manuf. Technol. 4, 372 (1981).

${ }^{4}$ G. Yu, M. Q. Zhang, and H. M. Zeng, J. Appl. Polym. Sci. 70, 559 (1998).

${ }^{5}$ R. Strümpler and J. Glatz-Reichenbach, J. Electrocerm. 3, 329 (1999).

${ }^{6}$ M. Narkis, Z. Stein, and A. Ram, Polym. Eng. Sci. 21, 1049 (1981).

${ }^{7}$ S.J. Huang, J.-K. Lee, and C.-S. Ha, Colloid Polym. Sci. 282, 575 (2003).

${ }^{8}$ J. C. Huang, Adv. Polym. Technol. 21, 299 (2002).

${ }^{9}$ J. C. Dawson and C. J. Adkins, J. Phys.: Condens, Matter 8, 8321 (1996).

${ }^{10}$ O. Kazuyuki and N. Yoshihide, Jpn. J. Appl. Phys. 10, 99 (1971).

${ }^{11}$ L. Benguigui, J. Yacubowicz, and M. Narkis, I. Polym. Sci., Part B: Polym. Phys. 25, 127 (1987).

12. Balberg, D. Azulay, D. Toker, and O. Millo, Int. J. Mod. Phys. B 18, 2091 (2004).

${ }^{13}$ R. Strümpler, 3. Appl. Phys. 80, 6091 (1996).

${ }^{14}$ J. Daniels, K. H. Haerdtl, and R. Wernicke, Philips Tech. Rev. 38, 73 (1978).

${ }^{15}$ W. Heywang, Solid-State Electron. 3, 51 (1961).

${ }^{16}$ W. Heywang, J. Am. Ceram. Soc. 47, 484 (1964).

${ }^{17}$ B. Huybrechts, K. Ishizaki, and M. Takata, J. Mater. Sci. 30, 2463 (1995).

${ }^{18}$ D. C. Sinclair and A. R. West, J. Appl, Phys. 66, 3850 (1989).

${ }^{19}$ W. Heywang, J. Mater. Sci. 6, 1214 (1971).

${ }^{20}$ G. H. Jonker, Solid State Electron. 7, 895 (1964).

${ }^{21}$ M. Okano, Y. Watanabe, and S.-W. Cheong, Appl. Phys. Lett. 82, 1923 (2003).

${ }^{22}$ K. Asadi, D. M. de Leeuw, B. de Boer, and P. W. M. Blom, Nat. Mater. 7, 547 (2008),

${ }^{23}$ K. Asadi, H. J. Wondergem, R. S. Moghaddam, C. R. McNeill, N. Stingelin, B. Noheda, P. W. M. Blom, and D. M. de Leeuw, Adv. Funct. Mater. 21, 1887 (2011).

${ }^{24}$ I. Katsouras, K. Asadi, M. Li, T. B. van Driel, K. S. Kjaer, D. Zhao, T. Lenz, Y. Gu, P. W. M. Blom, D. Damjanovic, M. M. Nielsen, and D. M. de Leeuw, Nat. Mater. 15, 78 (2016).

${ }^{25}$ T. Furukawa, Phase Transitions 18, 143 (1989).
${ }^{26}$ Y. J. Park, I. S. Bae, S. J. Kang, J. Chang, and C. Park, LEEE Trans. Dielectr. Electr. Inswl. 17, 1135 (2010).

${ }^{27}$ M. Li, I. Katsouras, C. Piliego, G. Glasser, I. Lieberwirth, P. W. M. Blom, and D. M. De Leeuw, J. Mater. Chem. C 1, 7695 (2013).

${ }^{28}$ M. Li, N. Stingelin, J. J. Michels, M.J. Spijkman, K. Asadi, R. Beerends, F. Biscarini, P. W. M. Blom, and D. M. de Leeuw, Adv. Funct. Mater. 22, $2750(2012)$.

${ }^{29}$ H. Kodama, Y. Takahashi, and T. Furukawa, Ferroelectrics 203, 433 (1997).

${ }^{30}$ K. Tashiro and M. Kobayashi, Phase Transitions 18, 213 (1989).

${ }^{31}$ T. Yamada and T. Kitayama, J. Appl. Phys. 52, 6859 (1981).

${ }^{32} \mathrm{G}$. Teyssedre, A. Bernes, and C. Lacabanne, J. Polym. Sci, Part B: Polym. Phys. 33, 879 (1995).

${ }^{33}$ G. M. Su, E. Lim, E. J. Kramer, and M. L. Chabinyc, Macromolecules 48 , 5861 (2015).

${ }^{34}$ C. R. McNeill, K. Asadi, B. Watts, P. W. M. Blom, and D. M. de Leeuw, Small 6, 508 (2010).

${ }^{35}$ T. Lenz, D. Zhao, G. Richardson, I. Katsouras, K. Asadi, G. Glaßer, S. T. Zimmermann, N. Stingelin, W. S. C. Roelofs, M. Kemerink, P. W. M. Blom, and D. M. de Leeuw, Phys. Status Solidi A 212, 2124 (2015).

${ }^{36}$ K. Asadi, T. G. de Boer, P. W. M. Blom, and D. M. de Leeuw, Adv. Funct. Mater. 19, 3173 (2009).

${ }^{37}$ A. J. J. M. Van Breemen, J. L. Van Der Steen, G. Van Heck, R. Wang, V. Khikhlovskyi, M. Kemerink, and G. H. Gelinck, Appl. Phys. Express 7, 031602 (2014).

${ }^{38}$ L. Nougaret, H. G. Kassa, R. Cai, T. Patois, B. Nysten, A. J. J. M. van Breemen, G. H. Gelinck, D. M. de Leeuw, A. Marrani, Z. Hu, and A. M. Jonas, ACS Nano 8, 3498 (2014).

${ }^{39}$ V. Khikhlovskyi, R. Wang, A. J. J. M. van Breemen, G. H. Gelinck, R. A. J. Janssen, and M. Kemerink, J. Phys. Chem, C 118, 3305 (2014).

${ }^{40}$ V. Khikhlovskyi, A. J. J. M. van Breemen, J. J. Michels, R. A. J. Janssen, G. H. Gelinck, and M. Kemerink, J. Polym. Sci, Pant B: Polym, Phys, 53, 1231 (2015).

${ }^{41}$ M. A. Khan, U. S. Bhansali, D. Cha, and H. N. Alshareef, Adv. Funct. Mater. 23, 2145 (2013).

${ }^{42}$ M. Kemerink, K. Asadi, P. W. M. Blom, and D. M. de Leeuw, Organ. Electron. 13, 147 (2012).

${ }^{43}$ A. van Breemen, T. Zaba, V. Khikhlovskyi, J. Michels, R. Janssen, M. Kemerink, and G. Gelinck, Adv. Funct. Mater, 25, 278 (2015).

${ }^{44}$ K. Asadi, M. Li, N. Stingelin, P. W. M. Blom, and D. M. de Leeuw, Appl. Phys. Lett. 97, 193308 (2010).

${ }^{45}$ T. Lenz, M. Ghittorelli, F. S. Benneckendorf, K. Asadi, C. Kasparek, G. Glasser, P. W. M. Blom, F. Torricelli, and D. M. de Leeuw, Adv. Funct. Mater. 26, 5111 (2016).

${ }^{46}$ J. B. Enns and R. Simha, J. Macromol. Sci, Part B 13, 11 (1977). 